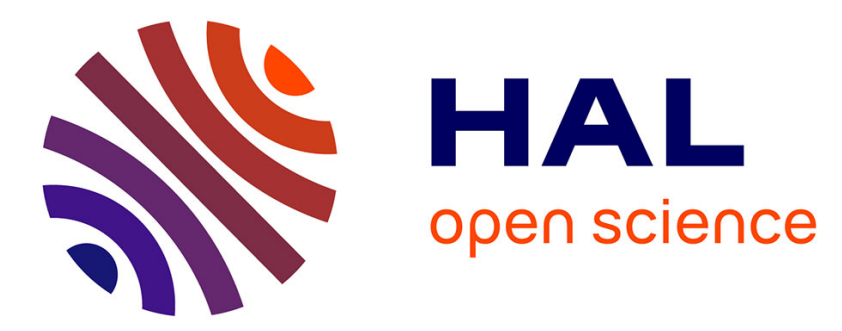

\title{
Self-centring technique for fibre optic microlens mounting using a concave cone-etched fibre
}

\author{
N.E. Demagh, A. Guessoum, R. Zeggari, T. Gharbi
}

\section{To cite this version:}

N.E. Demagh, A. Guessoum, R. Zeggari, T. Gharbi. Self-centring technique for fibre optic microlens mounting using a concave cone-etched fibre. Measurement Science and Technology, 2011, 22 (11), pp.115302. 10.1088/0957-0233/22/11/115302 . hal-00662994

\section{HAL Id: hal-00662994 \\ https://hal.science/hal-00662994}

Submitted on 6 May 2021

HAL is a multi-disciplinary open access archive for the deposit and dissemination of scientific research documents, whether they are published or not. The documents may come from teaching and research institutions in France or abroad, or from public or private research centers.
L'archive ouverte pluridisciplinaire HAL, est destinée au dépôt et à la diffusion de documents scientifiques de niveau recherche, publiés ou non, émanant des établissements d'enseignement et de recherche français ou étrangers, des laboratoires publics ou privés. 


\title{
Self-centring technique for fibre optic microlens mounting using a concave cone- etched fibre
}

\author{
Nacer-Eddine Demagh ${ }^{1}$, Assia Guessoum ${ }^{1}$, Rabah Zegari $^{2}$ and \\ Tijani Gharbi ${ }^{2}$ \\ ${ }^{1}$ Laboratoire d'Optique Appliquée, Institut d'Optique et de Mécanique de Précision, Université Ferhat \\ Abbas [Applied Optics Laboratory, Institute of Optics and Precision Mechanics, Ferhat Abbas \\ University], Sétif, Algeria \\ ${ }^{2}$ Laboratoire d'Optique P M Duffieux, FEMPTO-ST, Besançon, France \\ E-mail: ndemagh@yahoo.fr and ndemagh@univ-setif.dz
}

\begin{abstract}
Several techniques of centring a microlens onto the fibre optic end face are studied. In most of them, microsphere lenses are centred with the aid of high-accuracy micro-positioners. This process is complicated with regard to the difficulty in manipulating microsphere lenses. In this paper, a simple and accurate self-centring method for mounting microsphere lenses using a concave cone etched fibre (Demagh et al 2006 Meas. Sci. Technol. 17 119-22) is described. This technique allows the centring of a wide variety of microlens radii, typically $7 \mu \mathrm{m}$ to over $24 \mu \mathrm{m}$. The proposed process, however, is not affected by any spatial positioning control of microspheres. In over $85 \%$ of the attempts, the microsphere lenses were centred on the fibre axis to within $0.12 \mu \mathrm{m}$.
\end{abstract}

Keywords: self-centring, microsphere lens, micro-collimator, fibre optic, concave cone etched fibre, coupling efficiency

\section{Introduction}

The light power which can be launched into a single mode fibre (SMF) from a laser diode by simple butt coupling is small, being limited by the fibre acceptance angle. Centring of lenses on the fibre end is fundamentally important for purposes of increasing the light coupling efficiency. Thus, several coupling techniques have been studied $[1,2]$ where separate lenses are centred by means of very accurate cylindrical guides. Efficiency is also improved by the use of techniques based on adhesive surface tension [3]. Other collimator types involving GRIN lenses that are fused to the fibre are developed [4]. Because of the intrinsic eccentricity [5] and the small size of the fibre core, the microlens alignment procedure is more complex. It often requires a laser-light-assisted alignment technique. This process is complicated since it needs highaccuracy micro-positioners to manipulate microsphere lenses.

In order to prevent the complexity of a centring lenses procedure, microlenses are usually fabricated by melting the end of a tapered fibre to obtain a hemispheric microlens [6]. Besides, lenses are fabricated by coating the fibre end with a photoresist layer [7-9].

Among the proposed techniques separate microsphere lenses glued onto the fibre end face provide one of the highest coupling efficiencies. In fact, the latter arises in part from the ability to select lenses of a known radius and refractive index so as to approach optimal light coupling. Moreover, the highly spherical quality of such microspheres eliminates the type of curvature control problem involved in fabricating lenses at the fibre ends [10]. However, the small size of the core combined with the need to centre the lens accurately, typically within several micrometres, brings about difficulties in lens mounting.

The aim of this paper is to show that an intrinsic concave cone-etched fibre (CCEF) can be used in a self-centring microsphere lens technique. The centring procedure of the micro-ball is achieved without the need for any delicate 


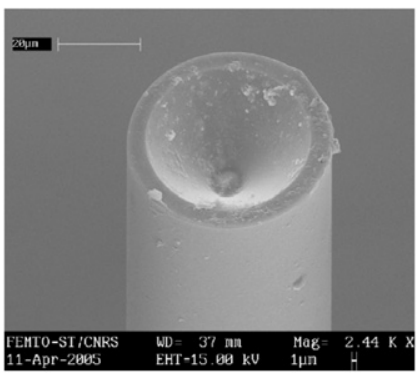

Figure 1. A scanning electron microscopy magnified view of a concave cone-etched end.
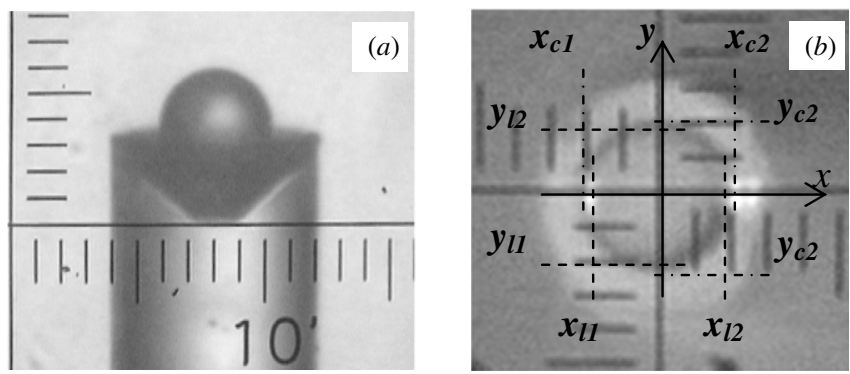

Figure 2. Profile view of a microsphere lens inserted into the concave cone end fibre $(a)$ and a front view of the inserted microlens (b).

centring apparatus. Thus, the technique can be described as self-centring.

\section{Materials and technique}

In a previous paper [11], we reported that a concave cone end fibre could be obtained chemically as opposed to the conventional fibre tip profile. Figure 1 shows a scanning electron microscopy magnified view of a typical concave cone fabricated at the end face of a SMF. This figure shows the principal parameters of the cavity: the vertex (core) and the internal and external boundaries of the base (cladding).

In optical fibres, the intrinsic eccentricity results in the core axis not being matched with the fibre axis regarding the external boundary of cladding. But, when a fibre is etched in HF medium, the core and the cladding are selectively etched in such a way that the core remains centred compared to the internal boundary of the base of the etched cone.

A microsphere lens of radius $r$ is then inserted into the concave cone as can be seen in figure 2. As a result, the microball lens slips automatically to the top of the cone. Thus, the ball lens remains centred with high accuracy close to the core axis.

By means of an optical microscope in the reflection mode, the images reflected from the lens as well as from the etched fibre are observed through the eyepiece of the autocollimator and projected on a CCD camera. Figure 2(a) illustrates a profile view of a $26 \mu \mathrm{m}$ diameter sphere lens inserted into the concave cone of the vertically handled etched fibre. A centring error can appear when the lens centre does not coincide with the fibre axis. Therefore, measurements of self-centring error are performed using the optical system of

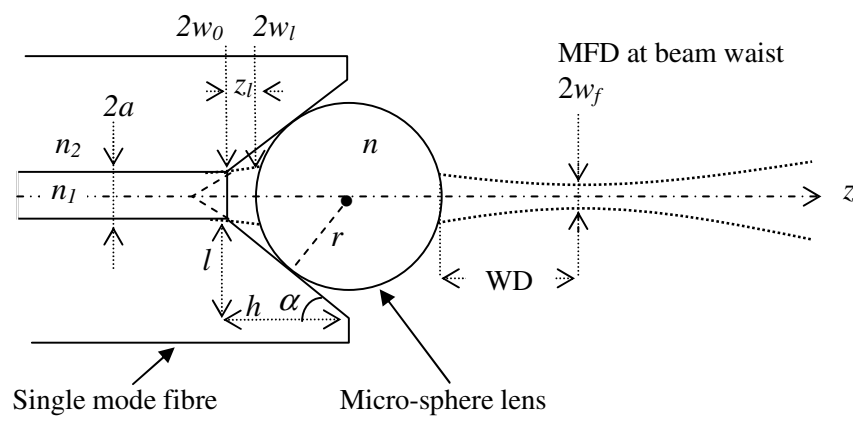

Figure 3. Representation of a concave cone micro-collimator construction.

Table 1. Mean and standard deviation of measurements in the two directions.

\begin{tabular}{lll}
\hline & Mean value $(\mu \mathrm{m})$ & Standard deviation $(\mu \mathrm{m})$ \\
\hline$\Delta x_{c}$ & 0.080 & 0.009 \\
$\Delta y_{c}$ & 0.121 & 0.008 \\
\hline
\end{tabular}

$0.02 \mu \mathrm{m}$ resolution. The procedure to make out the misalignment is to locate respectively the centre $\left(x_{l}, y_{l}\right)$ of the ball lens and the centre $\left(x_{c}, y_{c}\right)$ of the concave cone base by focusing the microscope both on the boundary of the lens and on the internal edge of the concave cone. The positions $\left(x_{l 1}\right.$, $\left.x_{l 2}\right),\left(y_{l 1}, y_{l 2}\right)$ of the lens and $\left(x_{c l}, x_{c 2}\right),\left(y_{c 1}, y_{c 2}\right)$ of the cone are fixed and measured successively in the two perpendicular directions $x$ and $y$, as illustrated in figure 2(b).

The point centre values of the lens and of the cone, respectively, are calculated as

$$
\begin{array}{ll}
x_{l}=\left(x_{l 1}+x_{l 2}\right) / 2 ; & y_{l}=\left(y_{l 1}+y_{l 2}\right) / 2 ; \\
x_{c}=\left(x_{c 1}+x_{c 2}\right) / 2 ; & y_{c}=\left(y_{c 1}+y_{c 2}\right) / 2
\end{array}
$$

and the misalignment in the two directions is evaluated by

$$
\Delta x_{c}=\left|x_{c}-x_{l}\right| \text { and } \Delta y_{c}=\left|y_{c}-y_{l}\right| .
$$

The experimental values are listed in table 1 .

We find $\Delta x_{c}=0.08 \mu \mathrm{m}$ and $\Delta y_{c}=0.12 \mu \mathrm{m}$. These values could be improved because most of the centring errors result from the measurement readout that depends on the tilt accuracy of the fibre axis shown in figure 2(a). It is obvious that the self-centring of the micro-ball is not affected by the tilt.

In the concave cone etching process, it is shown that a different concave cone size can be obtained since the cone height gradually extends as the etching proceeds until the concave cone boundary equals the etched diameter. In the particular case of our experimental interests, the value of the concave cone height $h$ is found to be $17.8 \mu \mathrm{m}$ and the concave cone base width $l$ is $15 \mu \mathrm{m}$. Consequently, a relatively large range of lens size can be mounted with desired cone dimensions. The radius range available can be derived from a simple geometry construction reported in figure 3 .

Let us consider on one hand that the minimum lens radius size $r_{\min }$ is the value which corresponds to the arrangement where the ball lens is tangential simultaneously to the cone wall and to the fibre core end face. On the other hand, the maximum value $r_{\max }$ is obtained when the ball lens is tangential 


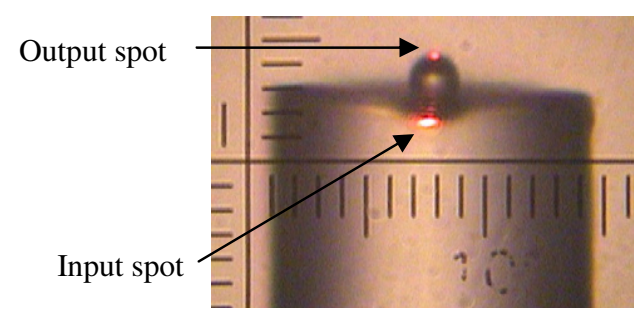

Figure 4. Representation of a magnified view of a typical micro-collimator.

(This figure is in colour only in the electronic version)

to the boundary line of the base at the maximum value of the height $h$. Thus, $r_{\min }$ and $r_{\max }$ can be expressed as

$$
\begin{array}{r}
r_{\min }=a \cos \alpha /(1-\sin \alpha) \\
r_{\text {max }}=(a+h \tan \alpha) / \cos \alpha,
\end{array}
$$

where $a$ is the core radius and $\alpha$ is the etched angle given by $\tan \alpha=l / h$. As a result, the radius range of microlenses that can be inserted in the concave cone is $r_{\min } \leqslant r \leqslant r_{\max }$.

With $a=3.5 \mu \mathrm{m}, l=15 \mu \mathrm{m}$ and $h=17.8 \mu \mathrm{m}$ ( $\alpha \sim 0.7 \mathrm{rad}$ ), we obtain $7.4 \mu \mathrm{m} \leqslant r \leqslant 24 \mu \mathrm{m}$.

Since the height of the CCEF [11] can be adjusted, as reported previously, a large assortment of collimators can be achieved. Figure 4 shows a micro-collimator that combines a polymer microsphere of a $4.5 \mu \mathrm{m}$ radius and a SMF $(4 / 125 \mu \mathrm{m})$ provided with a micro-cavity of $4 \mu \mathrm{m}$ height $(l \sim 3.5 \mu \mathrm{m})$.

\section{Theoretical analysis of propagated light}

Theoretically, the propagating light is assumed to have a Gaussian beam distribution. Therefore, the propagation characteristics of the Gaussian beam need to be taken into account. Figure 3 gives a description of the considered optical collimating device. As the fundamental mode of the SMF is approximated by a Gaussian radial distribution, the output light plane defined by the waist $2 w_{0}$ is projected on the microsphere input plane $2 w_{l}$. When the Gaussian light passes through the microlens, a new waist of a mode field diameter (MFD) $2 w_{f}$ is located at a working distance WD.

For a step index fibre, the mode field radius $w_{0}$ is given by the Marcuse formula [12] as follows:

$$
\frac{w_{0}}{a}=0.65+\frac{1.619}{V^{3 / 2}}+\frac{2.879}{V^{6}},
$$

where $a$ is the core radius and $V$ is the $v$-number given by

$$
V=\frac{2 \pi}{\lambda} a \sqrt{n_{1}^{2}-n_{2}^{2}},
$$

where $n_{1}$ and $n_{2}$ are respectively the core and the cladding refractive indices, $\lambda$ is the $\mathrm{He}-\mathrm{Ne}$ laser wavelength $(\lambda=$ $632.8 \mathrm{~nm})$ of the propagated beam and $\sqrt{n_{1}^{2}-n_{2}^{2}}$ is the numerical aperture $(\mathrm{NA}=0.21)$.

The Gaussian beam approximation for the fundamental mode helps in determining the beam parameters in any plane through a well-known formula. The equations used are
Table 2. Calculated parameters of the lenses of refractive index $n=1.4$.

\begin{tabular}{lrlll}
\hline & $z_{l}(\mu \mathrm{m})$ & $f(\mu \mathrm{m})$ & $w_{l}(\mu \mathrm{m})$ & $w_{f}(\mu \mathrm{m})$ \\
\hline Lens diameter $26 \mu \mathrm{m}$ & 4.8 & 22.7 & 2.2 & 1.5 \\
Lens diameter $50 \mu \mathrm{m}$ & 11.5 & 43.7 & 2.4 & 2.0 \\
\hline
\end{tabular}

familiar equations for the $1 / \mathrm{e}^{2}$ beam radius as a function of the distance $z$ from the waist, expressed by

$$
w_{l}(z)=w_{0} \sqrt{1+\left(\frac{z_{l}}{z_{0}}\right)^{2}} .
$$

In the Rayleigh range, the distance $z_{0}$ is given by

$$
z_{0}=\frac{\pi w_{0}^{2}}{\lambda}
$$

The distance $z_{l}$ propagated from the plane of $w_{0}$ where the wave front is flat to the lens input plane $w_{l}$ can be derived from figure 3 as

$$
z_{l}=r\left(\frac{1}{\sin \alpha}-1\right)-\frac{a}{\tan \alpha} .
$$

When the wave front $w_{l}$ of the Gaussian beam passes through the microsphere lens, a new waist $w_{f}$ is formed at an axial distance under paraxial assumption [13], assuming that the waist of an input beam represents the object and the waist of the output represents the image. The spot size can be determined by the following equation:

$$
w_{f}^{2}=\frac{w_{l}^{2}}{1+\left(\frac{\pi w_{l}^{2}}{\lambda}\right)^{2}\left(\frac{1}{f}-\frac{n}{R_{c}}\right)^{2}},
$$

where $R_{c}$ is the curvature radius of the wave front at the lens and $n$ is the refractive index of the microsphere lens.

From the optical theory, the effective focal length $f$ of a ball lens of radius $r$ is determined using the following equation:

$$
f \approx \frac{r}{2} \frac{n}{n-1} .
$$

The effective focal length is measured from the centre of the microsphere lens to its focal point. In reality, spherical aberration shifts the diffraction focus towards the microlens.

By assuming that the beam waist $w_{l}$ at the microlens input is an incident plane which is equivalent to having $R_{c}=$ $\infty$, equation (9) becomes

$$
w_{f}=\frac{w_{l}}{\left[1+\left(\frac{\pi w_{l}^{2}}{\lambda f}\right)^{2}\right]^{1 / 2}} .
$$

Substituting the experimental values in the preceding formulas, respectively (4)-(8), (10) and (11), the so-called parameters can be calculated. Interestingly, we find the spot size radius $w_{0}=2.21 \mu \mathrm{m}$, the mode field diameter $2 w_{0}=$ $4.42 \mu \mathrm{m}$, the $V$-number $V=2.385, z_{0}=24.24 \mu \mathrm{m}, z_{l}=$ $4.8 \mu \mathrm{m}$; at this distance, the wave front projected on the microball is assumed as plane $\left(R_{c}=\infty\right)$. The calculated parameters are listed in table 2.

The calculated parameters of the considered microcollimator depend on the micro-ball radius, its refractive index and the etched cavity size that fix the $z_{l}$ distance. 
However, before these characteristics can be optimized, the theoretical coupling efficiency must be considered. In fact, the optimum coupling occurs when both Gaussian beam and fibre beam waist sizes are matched. To express the optimal coupling, we introduce a coupling efficiency factor $\eta$.

From the Gaussian beam coupling theory [14], the theoretical calculation of the maximum butt jointing coupling efficiency $\eta$ and predicted improvement coupling efficiency $\eta(r)$ using lensed fibres can be expressed, respectively, as

$$
\eta=\frac{2 w_{s} w_{0}}{w_{s}^{2}+w_{0}^{2}}
$$

and

$$
\eta(r)=\frac{2 w_{s} w_{f}}{w_{s}^{2}+w_{f}^{2}},
$$

where $w_{s}$ is the waist of the focused Gaussian beam (figure 6).

Given $\eta$ and $\eta(r)$, one can calculate the ratio $\eta(r) / \eta$ of the increase in the coupling efficiency.

Using a microscope objective lens $(\times 20$ Plan Fluorite $)$ the waist diameter $2 w_{s}$ is found to be $2.13 \mu \mathrm{m}$. The microlens $(r=25 \mu \mathrm{m})$ was found to have a waist diameter of $2.02 \mu \mathrm{m}$. As a result, the increased ratio in coupling efficiency is then $\eta(r=25 \mu \mathrm{m}) / \eta \approx 1.2$.

The increase in the coupling efficiency $\eta(r)$ as a function of the inserted microlens radius $r$ is expected because the potential coupling efficiency exhibits an optimal value for a giving angle of CCEF (or the $z_{l}$ distance, equation (8)), and we can thus calculate the optimum radius of curvature for the microlens. Substituting (6), (8) and (11) into (13), the coupling efficiency $\eta(r)$ can be written as

$\eta(r)=2 w_{s} w_{0} \frac{\left\{(r A+B)\left(1+\frac{M^{2}}{r^{2}}(r A+B)^{2}\right)\right\}^{1 / 2}}{w_{s}^{2}\left(1+\frac{M^{2}}{r^{2}}(r A+B)^{2}\right)+w_{0}^{2}(r A+B)}$,

where

$$
\begin{gathered}
A=\frac{\lambda}{\pi w_{0}^{2}}\left(\frac{1}{\sin \alpha}-1\right) ; \quad B=\frac{-a}{\tan \alpha}\left(\frac{\lambda}{\pi w_{0}^{2}}\right)+1 ; \\
M=\frac{2(n-1)}{n} \frac{\pi w_{0}^{2}}{\lambda} .
\end{gathered}
$$

As stated above, the optimum coupling efficiency is dependent on the microlens radius. Figure 5 shows the coupling efficiency versus different radii of the microsphere lens.

At the optimal lens radius, the propagated beam's waist radius is close to the fibre's one, thus resulting in a maximum coupling efficiency. The optimal radius $r_{\mathrm{op}}$ is found to be $11.11 \mu \mathrm{m}$.

Otherwise, on the one hand, the coupling inefficiency is attributed in part to spherical aberration of lenses. The reduced coupling inefficiency is achieved with lenses of small radius and high refractive index, because the losses due to spherical aberration decrease with decreasing focal length. On the other hand, coupling loss increases more rapidly as the lens comes close to the fibre $\left(r \ll r_{\text {op }}\right)$ than as it moves in the opposite direction [15]. Therefore, a moderate coupling efficiency can be maintained with appropriate microlens radius.

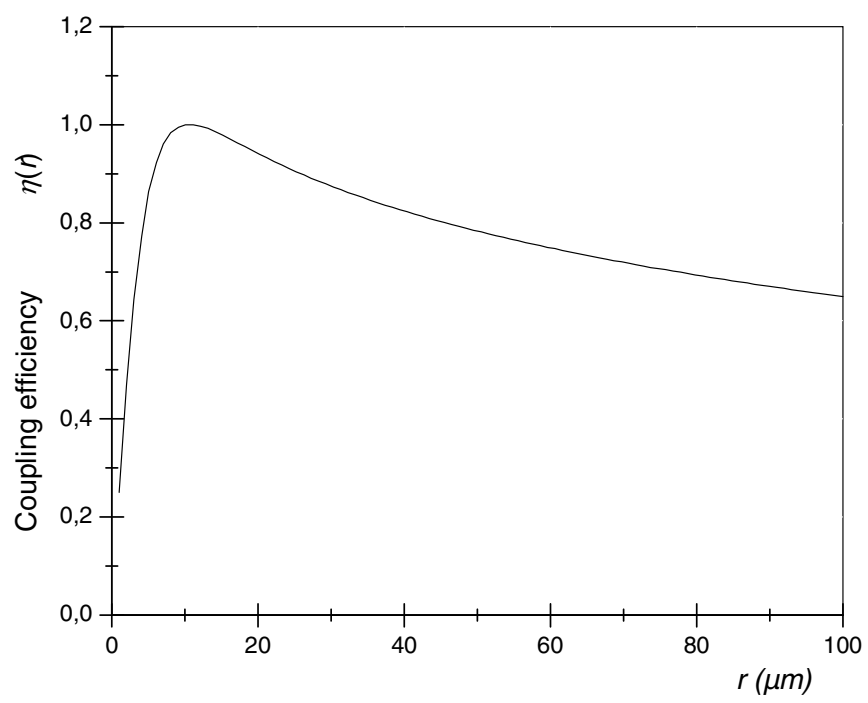

Figure 5. Coupling efficiency versus microlens radius.

\section{Coupling efficiency}

A coupling lens is an effective solution to provide the ability to easily launch the light from the divergent output of an optical device to a SMF. A critical issue is the coupling efficiency dependence of the coupling lens arrangement and misalignment tolerance. Therefore, in this section a comparison of measurements between the conventional coupling design and the proposed design was made with respect to light coupling.

Because the adjustment of the SMF can affect the initial alignment of the fibre and both the light source and the photodetector, the same fibre is used in the experimental setup for the two kinds of coupling design, successively without and with a mounted microlens to achieve an accurate comparison of the two coupling designs.

Beforehand, a SMF fibre $(4 / 125 \mu \mathrm{m})$ is etched as mentioned in the previous section, and then it is fixed in the measurement setup by mechanical multi-axis positioning stages as shown in figure 6 .

The alignment of the fibre is performed by means of precision translation stages in the lateral direction $(x, y)$ to achieve the best efficiency of optical power transfer between the focused light $(\lambda=632.8 \mathrm{~nm})$ and the optical fibre.

In the longitudinal direction $z$, a linear driven motorized stage, with a micrometer resolution over $25 \mathrm{~mm}$ displacement range, translates the fibre end in a dynamic range $\Delta z=$ $2.3 \mathrm{~mm}$ around the waist $w_{s}$ location plane $(z=0)$ of the injection source. The value of original transmitted power $P_{0}(z)$ as a function of the longitudinal displacement $z$ is measured.

In the microlens coupling design, a microball lens is inserted in the concave cone of the SMF. As a result, the photodetector (Si photodiode/0.3-1.1 $\mu \mathrm{m}$ ) gives the value of the transmitted light power $P_{l}(z)$ measured in the same dynamic range. Then, the coupling efficiency caused by the transferred light through the microlens can be calculated.

Two coupling sizes of microlens of $26 \mu \mathrm{m}$ and $50 \mu \mathrm{m}$ diameters are tested successively. The relevant experimental 


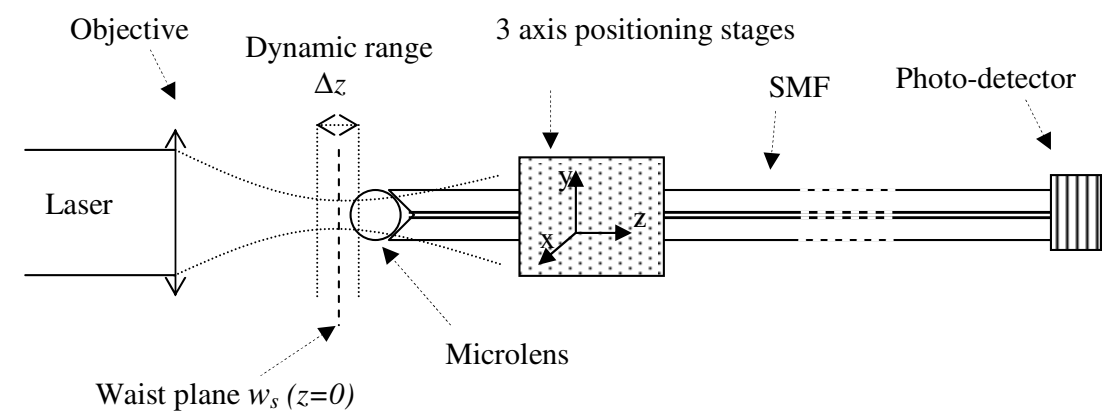

Figure 6. Experimental setup for coupling light measurements as the fibre end is translated in the longitudinal direction $z$ with and without a microlens.

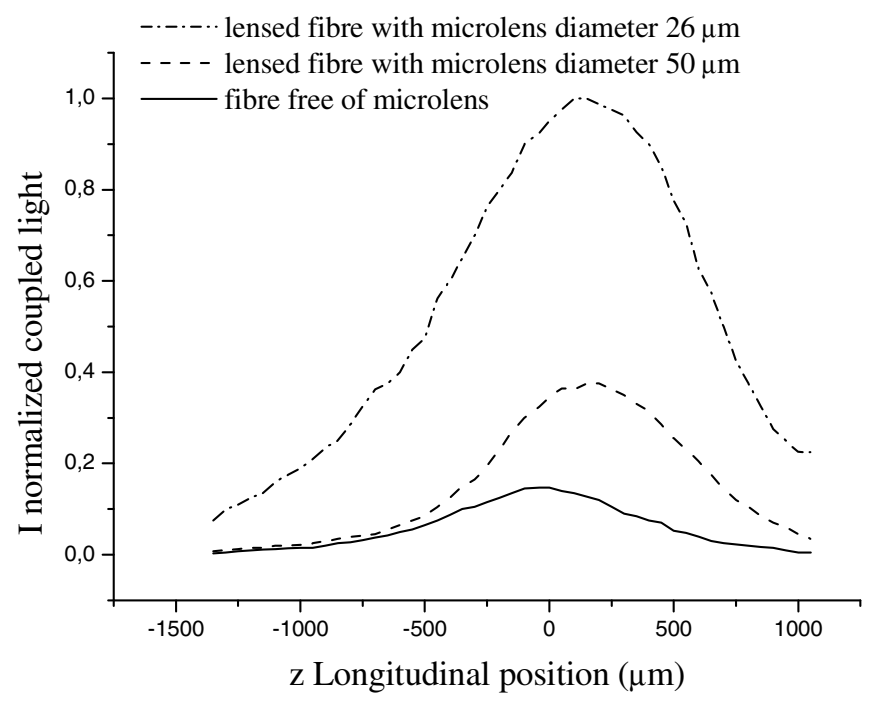

Figure 7. Normalized coupled light versus translated end fibre in the waist region of the objective focused light.

results of transmitted light are plotted in figure 7. The latter shows, at its best overall $(z=0)$, that the efficiency obtained in the lensed fibre design is improved by $44 \%$ (factor 2.5 ) with $50 \mu \mathrm{m}$ lens diameter and by $74 \%$ (factor 7) with $26 \mu \mathrm{m}$ lens diameter as compared with the highest efficiencies which could be obtained with the conventional butt coupling light. Thus, the coupling efficiency is significantly increased from $-7.13 \mathrm{~dB}$ with $50 \mu \mathrm{m}$ lens diameter to $-2.62 \mathrm{~dB}$ with $26 \mu \mathrm{m}$ lens diameter.

The calculated optimal radius $r_{\mathrm{op}}(\sim 11.11 \mu \mathrm{m})$ is in reasonably good agreement with the experimental one, as shown in figure 7 . The latter shows that the lens of a radius of $13 \mu \mathrm{m}$ gives the best increase in coupling efficiency.

To achieve a compact device shown in figure 8 , the concave cone is glued with a thin layer of transparent adhesive where the mounted micro-ball lens is UV bonded. Besides, the adhesive of the refractive index $n_{3}$, which closely approximates that of both the fibre core and the microlens, acts as an indexmatching material that minimizes the two Fresnel reflections. The latter occurs when light passes from the microlens into the air gap $\left(n_{0}=1\right)$ and from the air gap into the fibre core. A quantitative analysis based on the Fresnel equations gives the optical transmittance representing the fraction of the incident power that gets transmitted into the fibre.
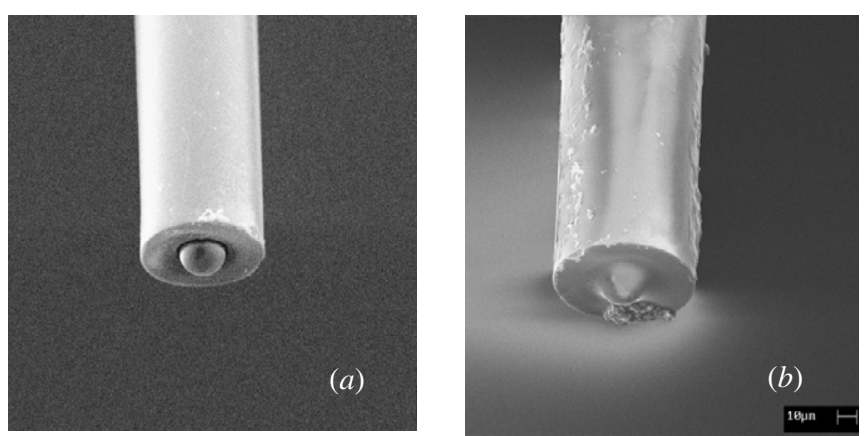

Figure 8. A scanning electron microscopy magnified view of a micro-collimator before $(a)$ and after $(b)$ bonding a $26 \mu \mathrm{m}$ microlens diameter onto 4/125 $\mu \mathrm{m}$ SMF.

Let $\rho_{10}$ and $\rho_{20}$ be the ratio of the reflected power to incident power of the two interfaces, and $\tau_{12}$ be the corresponding transmittance. Thus, we have the relationships $\rho_{10}=\left[\left(n_{1}-n_{0}\right) /\left(n_{1}+n_{0}\right)\right]^{2}, \quad \rho_{20}=\left[\left(n-n_{0}\right) /\left(n+n_{0}\right)\right]^{2}$,

$\tau_{12}=\left(1-\rho_{10}\right) \times\left(1-\rho_{20}\right)$.

The matching condition gives

$\rho_{13}=\left[\left(n_{1}-n_{3}\right) /\left(n_{1}+n_{3}\right)\right]^{2}, \quad \rho_{23}=\left[\left(n-n_{3}\right) /\left(n+n_{3}\right)\right]^{2}$,

$\tau_{13}=\left(1-\rho_{13}\right) \times\left(1-\rho_{23}\right)$.

Thus, the increase in transmission $\Delta \tau$ is accurately determined as $\Delta \tau=\left(\tau_{13}-\tau_{12}\right) / \tau_{12}$.

With $n_{3}=1.5$ and $n_{1}=1.4675$, the increase in this case would be over $6 \%$.

\section{Conclusion}

We have shown that a self-centring microsphere lens on a fibre optic end face can be achieved by inserting a micro-ball in a concave cone previously etched. In addition, a compact micro-collimator where the lens is UV bonded is presented.

Since the etching process allows us to obtain different dimensions of the cone height, a large range of lens size can be used to produce micro-collimators of desired optical parameters.

The above experiments are not limited only to those dimensions but lenses of radius greater than $r_{\max }(=24 \mu \mathrm{m})$ may be used. $r_{\max }$ depends, essentially, on the angle $\alpha$ which is determined experimentally for each step index fibre. 
Following this configuration, the lenses are disposed onto the concave cone edge where they remain self-centred.

In the case of figure 3 , the diameter of the fibre end remains relatively large $(\sim 38 \mu \mathrm{m}$ width), and so, by the etching process, it can be reduced to the micro-ball diameter in order to obtain a smaller micro-collimator.

Regarding the coupling efficiency, the proposed technique shows that inserted lenses were accurately centred on the fibre axis within $0.1 \mu \mathrm{m}$ rather than several micrometres as usually reported. This self-centring method which yields microlenses located to high accuracy produces a substantial improvement in the coupling efficiency. This efficiency increases by more than $74 \%$ in the compact configuration. Furthermore, the calculated lens radius is found to be in good agreement with the experimental value.

Because the centring microlens process is performed with no need for complicated positioning procedures as is the case of other techniques of mounting a separate lens (GRIN lens, C-lens, Ball-lens), the proposed method may provide a costcutting solution for micro-collimator applications and thus it can be appropriate for a production-oriented technique.

\section{Acknowledgments}

The authors are particularly grateful to A Malki from Rouen University (France), to L E Helseth from Florida State University (USA) and to R Louahdi from Setif University (Algeria). This work was supported by the Ministry of Higher Education and Scientific Research of Algeria (MESRS).

\section{References}

[1] Shiefman J 2004 Insertion loss comparison of microcollimators used to propagate light in and out of single-mode fibers Opt. Eng. 43 1927-37
[2] Tomas A and Markus R 2002 Chip-level integrated diffractive optical microlenses for multimode vertical-cavity surface-emitting laser to fiber coupling Opt. Eng. 41 3141-50

[3] Dakss M L and Kim B 1980 Simple self-centring technique for mounting microsphere coupling lens on a fiber Electron. Lett. 16 463-4

[4] Chanclou P, Kaczmarek C, Mouzer G, Gravey P, Thual M, Lecollinet M-A and Rochard P 2004 Expanded single-fiber using graded index multimode fiber Opt. Eng. 39 1634-42

[5] Shamir J, Shamir N and Karasikov N 1988 Nondestructive determination of core eccentricity in optical fibers by transverse scanning Opt. Eng. 27 587-93

[6] Malki A, Bachelot R and Van Lauwe F 2001 Two-step process for micro-lens-fibre fabrication using a continuous $\mathrm{CO}_{2}$ laser source J. Opt. A: Pure Appl. Opt. 3 291-5

[7] Hartmann D M, Reiley D J and Esener S C 2001 Microlenses self-aligned to optical fibers fabricated using the hydrophobic effect IEEE Photon. Technol. Lett. 13 1088-90

[8] Bear P D 1980 Microlens for coupling single-mode fibers to single-mode thin-film waveguides Appl. Opt. 19 2906-9

[9] Kim M-S, Jo K-W, Lee J-H, Song K-B, Kim E-K and Park K-H 2003 Self-aligned microlens fabricated on the sidewall of $45^{\circ}$ angled optical fiber for NSOM illumination system IEEE/LEOS Int. Conf. on Optical MEMS pp 18-9

[10] Barnard C W and Lit J W Y 1991 Single-mode fiber microlens with controllable spot size Appl. Opt. 30 1958-62

[11] Demagh N-E, Guessoum A and Aissat H 2006 Chemical etching of concave cone fibre ends for core fibres alignment Meas. Sci. Technol. 17 119-22

[12] Marcuse D 1977 Loss analysis of single-mode fiber splices Bell Syst. Tech. J. 56 703-18

[13] Ryu H-S and Kang H-S 2004 Analysis of wavelength effects on a spherical-end fiber lens Opt. Eng. 43 2212-3

[14] Kogelnik H 1964 Coupling and conversion coefficients for optical modes Proc. Symp. on Quasi-Optics (Brooklyn, NY: Polytechnic Press) pp 333-47

[15] Sumida M and Takemoto K 1984 Lens coupling of laser diodes to single-mode fibers J. Lightwave Technol. LT-2 305-11 